The system with zwitterionic lactose-based surfactant for complexation and delivery of small interfering ribonucleic acid-A structural and spectroscopic study

Michalina Skupin, Krzysztof Sobczak, Ryszard Zieliński, and Maciej Kozak'

Citation: Appl. Phys. Lett. 108, 213701 (2016); doi: 10.1063/1.4952589

View online: http://dx.doi.org/10.1063/1.4952589

View Table of Contents: http://aip.scitation.org/toc/apl/108/21

Published by the American Institute of Physics

A $1 \mathrm{P} \mid$ Applied Physics AlP Letters

Save your money for your research.

It's now FREE to publish with us no page, color or publication charges apply.

If your article has the

potential to shape the future of applied physics, it BELONGS in Applied Physics Letters 


\title{
The system with zwitterionic lactose-based surfactant for complexation and delivery of small interfering ribonucleic acid-A structural and spectroscopic study
}

\author{
Michalina Skupin, ${ }^{1}$ Krzysztof Sobczak, ${ }^{2}$ Ryszard Zieliński, ${ }^{3}$ and Maciej Kozak ${ }^{1,4, a)}$ \\ ${ }^{1}$ Department of Macromolecular Physics, Adam Mickiewicz University, Umultowska 85, 61-614 Poznań, \\ Poland \\ ${ }^{2}$ Department of Gene Expression, Adam Mickiewicz University, Umultowska 89, 61-614 Poznań, Poland \\ ${ }^{3}$ Department of Technology and Instrumental Analysis, Faculty of Commodity Science, Poznań University \\ of Economics, al. Niepodległości 10, 61-875 Poznań, Poland \\ ${ }^{4}$ Joint SAXS Laboratory, Adam Mickiewicz University, Umultowska 85, 61-614 Poznań, Poland
}

(Received 13 January 2016; accepted 13 May 2016; published online 24 May 2016)

\begin{abstract}
Systems suitable for the effective preparation of complexes with siRNA (small interfering RNA) are at the center of interest in the area of research work on the delivery of the RNA-based drugs (RNA-therapeutics). This article presents results of a study on the structural effects associated with siRNA complexation by a surfactant comprising a lactose group (N-(3-propanesulfone)-N-dodecylamino-beta-D-lactose hydrochloride, LA12). The double stranded siRNA oligomer (21 base pairs) used in this study is responsible for silencing a gene that can be important in the therapy of myotonic dystrophy type 1 . The obtained siRNA/LA12 lipoplexes were studied using the methods of small angle scattering of synchrotron radiation, circular dichroism spectroscopy, Fourier transform infrared spectroscopy, and electrophoretic mobility tests. Lipoplexes form in solution stable lamellar or cubic phases. The surfactant selected for the study shows much lower cytotoxicity and good complexation abilities of siRNA than dicationic or polycationic surfactants. Published by AIP Publishing.
\end{abstract}

[http://dx.doi.org/10.1063/1.4952589]

Recently, much attention has been paid to nucleic acid complexation with a number of chemically diverse ligands of potential medical applications. ${ }^{1-3}$ A large number of dicationic and polycationic complexing agents that effectively bind and stabilise nucleic acids and thus can be used in gene therapy ${ }^{4,5}$ or in transfer of therapeutic drugs based on siRNA or other short nucleic acids have been described. ${ }^{6,7}$ The search for new delivery systems is aimed at development of such compounds that would not only be effective in complexation of nucleic acids, especially siRNA (small interfering RNA), but will also show the lowest possible cytotoxicity. Interesting agents, promoting the formation of condensed forms of nucleic acids, are zwitterionic surfactants with saccharide moiety (sugar-based surfactants). They make a promising group of compounds capable of complexing nucleic acids and of formation of different spatial forms. ${ }^{8-10}$

This paper reports results of our study on the effect of complexation by a surfactant comprising a lactose group (N-(3-propanesulfone)-N-dodecyl-amino-beta-D-lactose hydrochloride, LA12) on the conformation of a short double stranded siRNA oligomer (21 base pairs). The sequence studied is responsible for silencing the gene that can be important in therapy of myotonic dystrophy type 1 (DM1). ${ }^{11-13}$

DM1 is the most frequent form of muscular dystrophy in adults. ${ }^{14}$ It is an autosomal-dominant hereditary disease which can affect many tissues and produce diverse symptoms, including muscle hyperactivity (myotonia), progressive myasthenia,

\footnotetext{
${ }^{\text {a) }}$ Author to whom correspondence should be addressed. Electronic mail: mkozak@amu.edu.pl
}

heart conduction defects, cardiomyopathy, insulin resistance, or neuropsychological disturbances. ${ }^{14,15}$ DM1 is caused by the expansion of trinucleotide CTG repeats in $3^{\prime}$ UTR $\left(3^{\prime}\right.$ untranslated region) of DM protein kinase gene (DMPK). The main feature of DM1 pathogenesis is production of toxic RNA containing hundreds or thousands of CUG repeats, which is accumulated in the nucleus. This leads to incorrect alternative splicing of many specific pre-mRNA through a change in activity of two families of splicing factors, namely, MBNLs (Muscleblind-like proteins) and CUGBPs (CUG binging proteins). ${ }^{16}$ MBNLs are less active due to their sequestration on expanded CUG repeat RNA and the activity of CUGBP1 protein increases due to its hyper-phosphorylation. Therefore, prevention of the diseases would be achieved by normalization of activity of these proteins using different antisense strategies. ${ }^{16}$ One of the possible ways to downregulate CUGBP1 expression is to use RNA interference tools such as small interfering RNAs. The main problem of this strategy is low stability of siRNA and lack of efficient delivery systems of these reagents. ${ }^{12,13}$ The complex proposed in our study should effectively bind and stabilise siRNA for transfection and should show low toxicity for a variety of cells. For the lactose based surfactants chosen (LA12), the bonding of lactose moiety with the hydrophobic fragment via an amine group ensures effective proton bonding in acidic environment, which means that they can act as cationic surfactants.

To characterise the ability of studied surfactant to form stable complexes with siRNA and to get information on the structures of complexes formed and processes of their selfassembly, we applied circular dichroism (CD) and Fourier transform infrared (FTIR) spectroscopies, small angle X-ray 
scattering (SAXS), and electrophoretic tests. Moreover, the surfactant was subjected to cytotoxicity tests as this property is essential for applications in gene therapy.

Selected for this study the double-stranded siRNA oligomer (antisense:5'-pGAAACAGGAUUGUCGUAACdTdT-3', sense: $5^{\prime}$-pGUUACGACAAUCCUGUUUCdTdT- $3^{\prime}$ ) has the nucleotide sequence that blocks CUGBP1 protein biosynthesis. The siRNA samples (FutureSynthesis, Poznań, Poland) were directly dissolved in $10 \mathrm{mM}$ sodium phosphate buffer $\mathrm{pH}$ 7.0. The surfactant (N-(3-propanesulfone)-N-dodecyl-aminobeta-D-lactose hydrochloride, LA12) was prepared as described previously. ${ }^{17}$ Complexes of siRNA and the LA12 surfactant were prepared by directly mixing the siRNA solution with surfactant solutions and incubated $(\sim 15 \mathrm{~min})$ at room temperature prior to taking spectroscopic or SAXS measurements. A series of lipoplexes with the LA12 surfactant concentration from 2 to $200 \mathrm{mM}$ ( $\mathrm{p} / \mathrm{n}$ charge ratio: 1-100) was prepared. SAXS data (q-axis range 0.12 to $4.6 \mathrm{~nm}^{-1}$, where $\left.\mathrm{q}=4 \pi \sin \theta / \lambda, \lambda=0.15 \mathrm{~nm}\right)$ were collected on the P12 beamline ${ }^{18,19}$ at the Petra-III storage ring (DESYHamburg, Germany). The SAXS data were processed and analysed using PRIMUS program. ${ }^{20,21}$ The circular dichroism (CD) spectra for the reference siRNA solution and siRNA/LA12 lipoplexes were collected using a J-815 spectropolarimeter (Jasco, Japan). The CD measurements (the spectral range from $200 \mathrm{~nm}$ to $320 \mathrm{~nm}$, at a $50 \mathrm{~nm} / \mathrm{s}$ scanning speed and $1 \mathrm{~nm}$ band-width) were conducted at room temperature in quartz cuvettes with a $0.5 \mathrm{~mm}$ beam path. Electrophoretic mobility tests of studied lipoplexes were performed using standard conditions $(120 \mathrm{~V}$ for $60 \mathrm{~min})$ and procedures in agarose gels $(1.5 \% \mathrm{w} / \mathrm{w})$. The reference samples were DNA marker (Sigma-Aldrich) and siRNA solution. The electrophoretic mobility patterns were visualised using a standard $300 \mathrm{~nm}$ UV transilluminator. The infrared spectra (spectral range of $4000-600 \mathrm{~cm}^{-1}$, resolution $4 \mathrm{~cm}^{-1}$ ) for siRNA/LA12 lipoplexes were recorded by the use of the attenuated total reflection technique and Tensor-27 (Bruker-Optics, Ettlingen, Germany) spectrometer. Cultures of HeLa cell lines and fibroblasts (GM04033 and GM07492 cell lines) were grown in the $\mathrm{CO}_{2}$ atmosphere (5\%). For the culture of HeLa cells, Dulbecco's Modified Eagle's medium (DMEM) (Lonza, Switzerland) with Lglutamine supplemented with $10 \%$ FBS, $1 \%$ mixture of antibiotics, and antimycotic agents was used, while for the culture of the fibroblast cells Eagle's Minimal Essential medium (EMEM) (Lonza, Switzerland) was used. To evaluate the cytotoxicity of LA12 surfactant, the cell cultures (80\% confluency) were treated with various concentrations of LA12. After $1 \mathrm{~h}$ of incubation, the cells were observed using a light microscope, and then a colorimetric $3-(4,5-$ dimethylthiazol-2-yl)-2,5-diphenyltetrazolium bromide (MTT) assay was performed, which is the cellular reduction of 3-(4,5-dimethylthiazol-2-yl)-2,5-diphenyltetrazolium bromide (MTT) (Cayman-Chemical, Michigan, USA), according to the manufacturer's instructions. ${ }^{22}$

The CD spectra obtained for the series of complexes analysed in this study are presented in Fig. 1(a). The reference CD curve recorded for pure siRNA solution provides unambiguous evidence of the presence of A-RNA in this solution. Complexation of siRNA with the surfactant
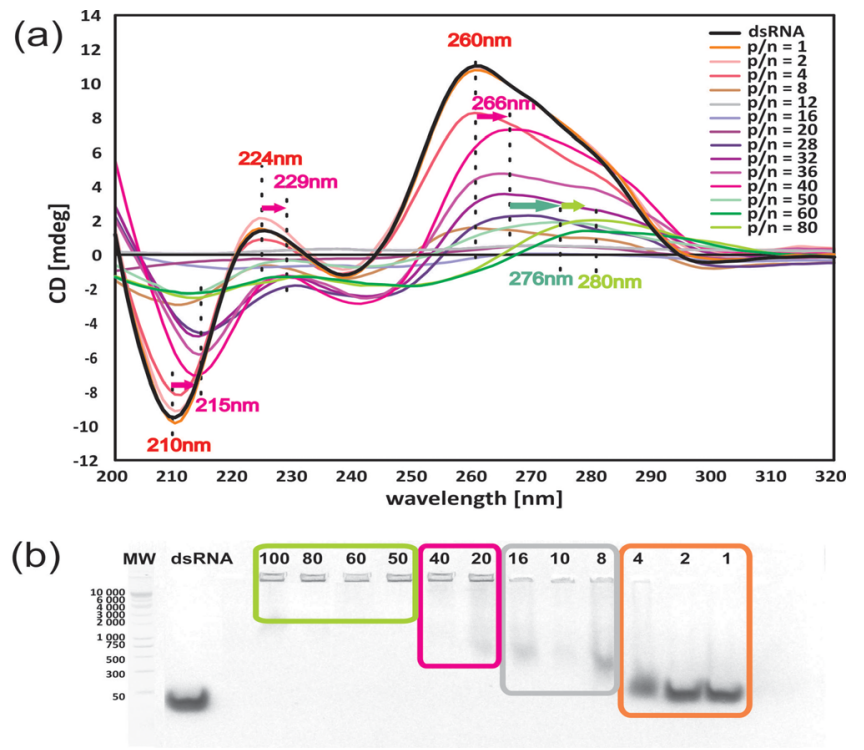

FIG. 1. The CD spectra recorded for the reference siRNA solution and LA12/siRNA complexes $(\mathrm{p} / \mathrm{n}=1-80)$. The shifts in CD spectra were marked by arrows (a). Results of electrophoretic mobility tests of LA12/siRNA lipoplexes $(\mathrm{p} / \mathrm{n}=1-100)$ and synthetic siRNA oligomer (b). The first lane is the molecular weight marker. Subsequent lipoplexes are marked according to the observed in CD spectra changes.

studied, in concentration of $16 \mathrm{mM}$ in solution (the charge ratio $\mathrm{p} / \mathrm{n}=8$, where $\mathrm{p} / \mathrm{n}$ is defined as the positive-to-negative electric charge ratios of lipoplexes), leads to a drastic decrease in the intensity of the CD signal of A-RNA. A substantial decrease in the intensity of the bands characteristic of A-RNA ${ }^{23}$ indicates increasing contribution of the interactions between the second and the third neighbours, leading to local disturbances in the geometry of nucleic bases. A consequence of these disturbances can be the process of siRNA condensation in the complex. For the systems with higher surfactant concentrations, no CD signal was detected. The complexation was confirmed by electrophoretic studies, allowing observation of a small amount of RNA moving in the gel at the ratio $\mathrm{p} / \mathrm{n}=8$. On further increasing concentrations of the surfactant, from its certain value a total arrest of electrophoretic mobility of siRNA was observed, which means that the RNA charge was nullified by the surfactant. After addition of higher amounts of the surfactant to siRNA solution $(\mathrm{p} / \mathrm{n}=28-40)$, the CD signal was restored and the A-RNA spectrum shift towards longer wavelengths was observed. The opaque white solution again became transparent and clear. The shift of the bands (maximum and minimum) can indicate the interaction between the positively charged groups of the surfactant polar head group showing properties of a cationic surfactant, and the polyanionic RNA molecule.

Electrophoretic measurements confirm the changes, RNA shows intense fluorescence close to the gel loading wells $(\mathrm{p} / \mathrm{n}=20)$ (Fig. 1(b)), which is the evidence of nullification of charges of polyanionic RNA by the surfactant. It is a result of the exposition of the hydrophobic parts of the surfactant to the solution and an increase in polarity of the local environment of chromophores in the molecule of siRNA. An additional change in the spectrum is a broadening of the positive band showing two components. The broadening of the positive band and its shift towards longer wavelength has 
been observed earlier and assigned to the intermediate form between the forms A and Z. ${ }^{24}$

This intermediate form was labelled as $Z^{\prime}$, and its characteristic feature is a significant shift of its $C D$ spectrum towards longer wavelengths. With increasing surfactant concentration, the observed ellipticity decreases and the characteristic bands are shifted towards longer wavelengths, which is the evidence of increasing efficiency of interactions of the acid with the surfactant and the formation of stable complexes that can enhance the effectiveness of transfection and penetration into cells. The stable complexes protect the RNA against nucleases and facilitate penetration through the small openings in the cell membrane.

The structure and symmetry of the complexes forming in the solution were determined by the SAXS measurements. The SAXS curves for the surfactant and the siRNA complexes are presented in Fig. 2. For the lowest concentrations of the surfactant $(2 \mathrm{mM}$ and $4 \mathrm{mM})$, the SAXS curve shows individual peaks that can be assigned to the formation of a single lamellar phase. After addition of siRNA to the surfactant solution, no significant changes in the curve were noted.

For higher LA12 surfactant concentrations $(8 \mathrm{mM}, 16 \mathrm{mM}$, and $20 \mathrm{mM}$ ), the SAXS curves of the reference systems show double diffraction peaks that evidence the coexistence of two lamellar phases. After addition of siRNA, besides the diffraction peaks assigned to the two lamellar phases, there was a signal of increasing intensity which is interpreted as corresponding to micelles forming in the solution.

The SAXS results can be correlated with electrophoretic analysis of the process of complexation. With increasing concentration of the surfactant, the signal assigned to micelles increases as does the intensity of diffraction peaks coming from the lamellar phases, and the progress in complexation is accompanied by cessation of electrophoretic mobility and disappearance of the CD signal of siRNA, which evidences the siRNA binding in complexes with the surfactant. The SAXS curve recorded for the reference solution of the surfactant in concentration of $32 \mathrm{mM}$ indicates the presence of a lamellar phase. The SAXS curve obtained for the system with siRNA complexes with the surfactant present in the same concentration shows an intense signal from the micellar phase and lamellas formed by the surfactant. In the solutions of surfactants at higher concentrations, the formation of liposomal structures coexisting with the lamellar phase was observed. In the systems with siRNA and the surfactant at the same concentrations, the SAXS results indicate the formation of ordered structures whose diffraction maxima prove the dominance of the cubic phase. For the reference solutions with higher concentrations of the surfactant (above $40 \mathrm{mM}$ ), the micellar phase is dominant. In the systems with siRNA and surfactants in the same concentrations, the SAXS data provide evidence of the presence of cubic phase $\left(\mathrm{a}_{0}=4.47-4.52 \mathrm{~nm}\right)$ coexisting with the lamellar phase $\left(\mathrm{d}_{001}=3.28 \mathrm{~nm}\right)$. For these systems, the CD data show the restoration of the A-RNA spectrum and a characteristic shift towards longer wavelengths. The above presented results following from the studies of the siRNA and surfactant complexation process indicate formation of stable complexes and partial exposition of the hydrophobic fragments of the surfactant towards the solution, which permits the appearance of ordered cubic phases in the solution.

To confirm the conformation of siRNA obtained from CD measurements, a complementary FTIR study was performed. Studied siRNA in solution assumes the form of A-RNA, which is confirmed by the FTIR spectrum ${ }^{25,26}$ (Fig. 3), showing characteristic absorption bands at $811 \mathrm{~cm}^{-1}, 864 \mathrm{~cm}^{-1}, 916 \mathrm{~cm}^{-1}$, and $1244 \mathrm{~cm}^{-1}$. In the presence of the surfactant, the FTIR spectra of siRNA above the surfactant concentration of $16 \mathrm{mM}$ $(\mathrm{p} / \mathrm{n}=8)$ reveal the disappearance of the characteristic bands at $811 \mathrm{~cm}^{-1}$ and $864 \mathrm{~cm}^{-1}$. It means that at higher surfactant concentrations, the RNA helix can be partly unfolded. ${ }^{27}$ The unfolding can be related to lower elasticity of A-RNA (in contrast to A-DNA). ${ }^{28}$

Further evidence of changes in the siRNA structure is the presence of bands assigned to the vibrations of phosphatesugar backbone $\left(800-840 \mathrm{~cm}^{-1}\right)$ and the shifts of these bands. The FTIR spectra reveal changes in the intensities (Fig. S1 ${ }^{37}$ ) of the bands at $811 \mathrm{~cm}^{-1}, 864 \mathrm{~cm}^{-1}$, and $876 \mathrm{~cm}^{-1}$, which are markers of the ribose fragments in the N-type conformation $\left(\mathrm{C}^{\prime}\right.$-endo/anti) in nucleic acids ${ }^{25,29,30}$ and the shifts of the bands in the range $823-826 \mathrm{~cm}^{-1}$ accompanying the increase in the surfactant concentration (the ribose S-conformation, $\mathrm{C} 2$-endo/anti). The changes in FTIR spectra observed with increasing surfactant concentration evidence a direct influence (a)

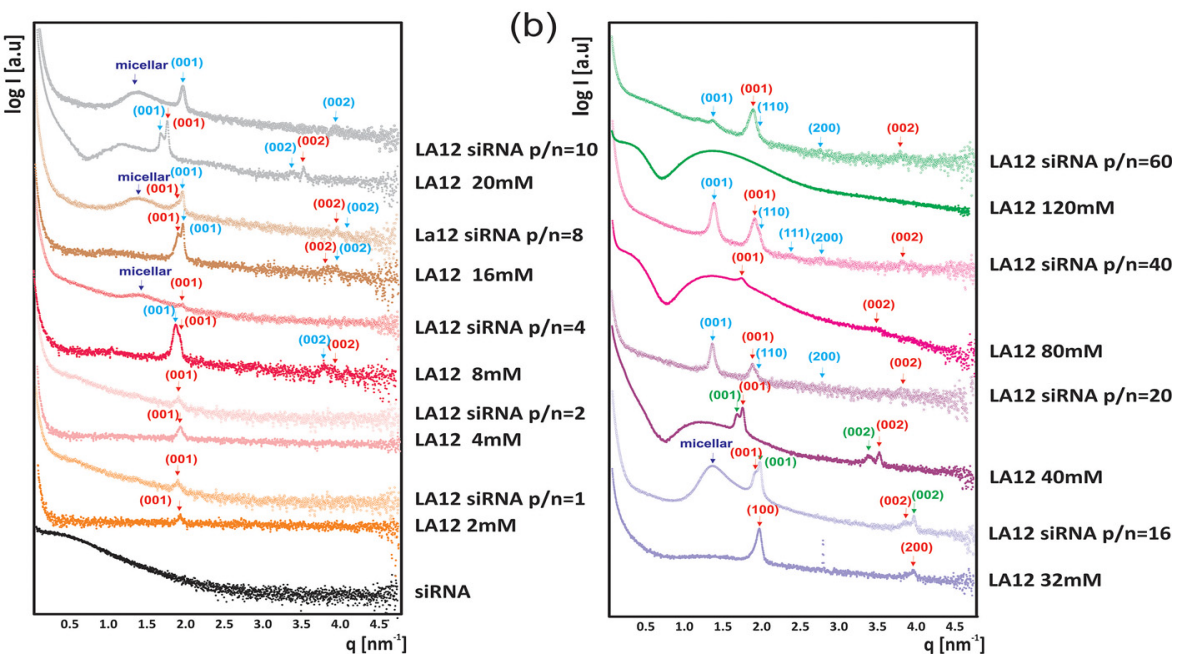

FIG. 2. The SAXS data obtained for LA12/siRNA lipoplexes and corresponding reference surfactant solutions in surfactant concentration range from 2 to $20 \mathrm{mM}(\mathrm{p} / \mathrm{n}=1-10)$ (a) and from 32 to $12 \mathrm{mM}(\mathrm{p} / \mathrm{n}=16-60)$ (b). Subsequent lipoplexes are marked in colours according to $\mathrm{CD}$ spectra. 


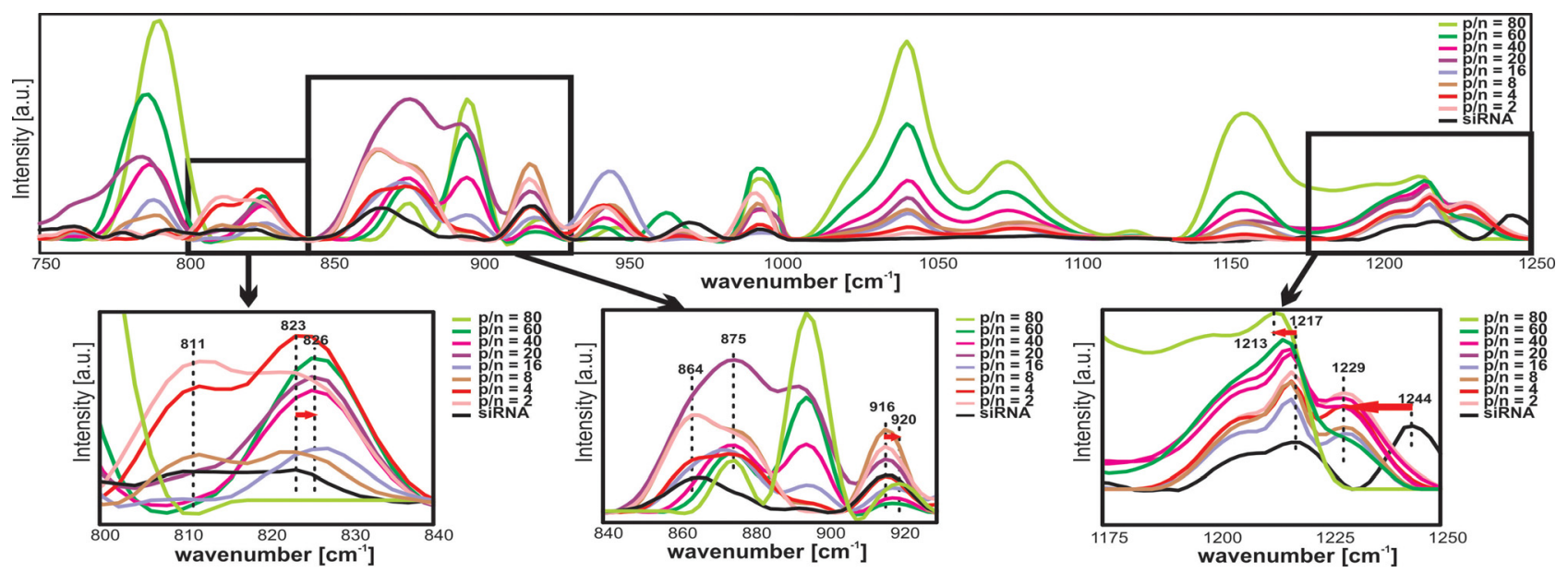

FIG. 3. FTIR spectra collected for LA12/siRNA lipoplexes and magnifications at characteristic absorption bands.

of the surfactant on ribose conformation. Increase in the surfactant concentration also causes shifts of the bands at $916 \mathrm{~cm}^{-1}, 970 \mathrm{~cm}^{-1}$ (Fig. $\mathrm{S1}^{37}$ ), and $995 \mathrm{~cm}^{-1}$ in the FTIR spectra towards longer wavenumbers, which can be a result of the interaction of surfactant with $2^{\prime}-\mathrm{OH}$ groups of the nucleic acid or ribose..$^{30-32}$ For A-RNA in solution, the characteristic bands at $1244 \mathrm{~cm}^{-1}$ and $1217 \mathrm{~cm}^{-1}$ are assigned to the stretching asymmetric vibrations of $\mathrm{PO}_{2}$ groups. ${ }^{25,30,32,33}$ With increasing surfactant concentration, the band $1217 \mathrm{~cm}^{-1}$ is shifted towards shorter wavenumbers which may indicate disturbance of A-RNA structure (Fig. $\mathrm{S}^{37}$ ). A similar shift has been observed on transformation of A-DNA into Z-DNA ${ }^{34}$ and in RNA study in acidic environment. ${ }^{35}$ Other characteristic features in this range are the disappearance of the band at $1244 \mathrm{~cm}^{-1}$ and the appearance of a new band at $1229 \mathrm{~cm}^{-1}$. Such a behaviour indicates the earlier postulated partial unfolding of the RNA helix and its local changes. ${ }^{36}$

In parallel to the structural study, the cytotoxic effect of the lactose surfactant on the cell cultures (HeLa and fibroblasts GM04033 and GM07492) was evaluated on the basis of qualitative (the cell morphology) and quantitative measurements (metabolic activity). The GM04033 cells were derived from DM1 patients having the expansion of about 1000 CTG repeats in the DMPK gene. The GM07492 fibroblasts were derived from healthy individual.

The microscopic observations performed for HeLa cells after $1 \mathrm{~h}$ of incubation revealed no significant changes in the morphology and number of cells in the presence of the surfactant in the concentrations from $0.625 \mu \mathrm{M}$ to $10 \mu \mathrm{M}$. The highest concentration of the LA12 surfactant added to HeLa cells was $20 \mu \mathrm{M}$, at which insignificant morphological changes and a decreased rate of cell growth were observed. When exposed to the surfactant at concentrations $40 \mu \mathrm{M}$ and $80 \mu \mathrm{M}$, the cells probably die, which was manifested by tearing off of the cells from the surface, the appearance of grains, and destruction of the cell membranes (Fig. S2 ${ }^{37}$ ).

For GM04033 fibroblasts, after $1 \mathrm{~h}$ incubation significant morphological changes were observed with increasing concentration of the surfactant. For LA12 concentrations 0.625-2.5 $\mu \mathrm{M}$, no morphological changes were observed, but a small decrease in the number of cells was noted, which can be caused by a decrease in the rate of their proliferation or adherence rate in the presence of the surfactant. ${ }^{37}$ In LA12 concentrations higher than $2.5 \mu \mathrm{M}$, the cells have rounded shape and tear off from the surface, but no significant morphological changes were observed. It can be also concluded that in the first phase of the experiment, the surfactant probably disturbed or blocked the elements of glycocalyx, i.e., the layer covering cell membrane from outside, built mainly of polysaccharide chains. After exposition to the surfactant in concentration of $40 \mu \mathrm{M}$, the cells were probably destroyed and washed out to such a degree that they were removed with the medium and surfactant. Similar behaviour was observed for the GM07492 cells.

As follows from the quantitative analysis (Fig. S2 ${ }^{37}$ ), the presence of surfactant in the cell culture medium has a significant effect on the number of life cells at high surfactant concentrations $(100 \mu \mathrm{M}-400 \mu \mathrm{M})$, at $\mathrm{EC}_{50}$ noted for $52.2 \pm 0.4 \mu \mathrm{M}$. At lower concentrations, below $14.1 \pm 0.4 \mu \mathrm{M}$ and even below $10 \%$, a small reduction in the number of life cells was observed, which can be a result of decreased rate of cell proliferation. In similar cytotoxicity tests on fibroblasts, $\mathrm{EC}_{50}$ was noted for $14.4 \pm 5.9 \mu \mathrm{M}$.

To sum up, the surfactant chosen for the study shows much lower cytotoxic'ity and good complexation abilities of siRNA when compared with dicationic surfactants, ${ }^{38}$ which means that it could be applied as a component of transfection systems in gene therapy.

This research project has been financed by the funds from the National Science Centre (Poland) granted on the basis of decision No. DEC-2011/01/B/ST5/00846 (M.S., M.K.) and Foundation for Polish Science TEAM program grant No. TEAM/2011-7/10 (K.S.).

${ }^{1}$ S. L. Gerson and E. C. Lattime, Gene Therapy of Cancer: Translational Approaches from Preclinical Studies to Clinical Implementation (Academic Press, 2002).

${ }^{2}$ M. Shichiri, A. Tanaka, and Y. Hirata, Gene Ther. 10(9), 827 (2003).

${ }^{3}$ M. Ogris, G. Walker, T. Blessing, R. Kircheis, M. Wolschek, and E. Wagner, J. Controlled Release 91(1-2), 173 (2003).

${ }^{4}$ V. D. Sharma, J. Lees, N. E. Hoffman, E. Brailoiu, M. Madesh, S. L. Wunder, and M. A. Ilies, Mol. Pharm. 11(2), 545 (2014). 
${ }^{5}$ N. Ernst, S. Ulrichskötter, W. A. Schmalix, J. Rädler, R. Galneder, E. Mayer, S. Gersting, C. Plank, D. Reinhardt, and J. Rosenecker, J. Gene Med. 1(5), 331 (1999).

${ }^{6}$ S. Falsini, S. Ristori, L. Ciani, E. Di Cola, C. T. Supuran, A. Arcangeli, and M. In, Soft Matter 10(13), 2226 (2014).

${ }^{7}$ R. Xu, X. Wang, and Z. R. Lu, Chin. Sci. Bull. 57(31), 3979 (2012).

${ }^{8}$ C. Bombelli, L. Giansanti, P. Luciani, and G. Mancini, Curr. Med. Chem. 16(2), 171 (2009).

${ }^{9}$ C. Stubenrauch, Curr. Opin. Colloid Interface Sci. 6(2), 160 (2001).

${ }^{10}$ L. Matthew and C. P. Fielden, Eur. J. Biochem. 268(5), 1269 (2001).

${ }^{11}$ G. Dujardin, E. Buratti, N. Charlet-Berguerand, M. M. Araujo, A. de Mbopda, C. L. Jossic-Corcos, F. Pagani, C. Ferec, and L. Corcos, Nucl. Acids Res. 38(20), 7273 (2010).

${ }^{12}$ N. A. Timchenko, Z. J. Cai, A. L. Welm, S. Reddy, T. Ashizawa, and L. T. Timchenko, J. Biol. Chem. 276(11), 7820 (2001).

${ }^{13}$ I. A. Vlasova, N. M. Tahoe, D. Fan, O. Larsson, B. Rattenbacher, J. R. SternJohn, J. Vasdewani, G. Karypis, C. S. Reilly, P. B. Bitterman, and P. R. Bohjanen, Mol. Cell 29(2), 263 (2008).

${ }^{14}$ S. R. Hammans, Brain 125(8), 1225 (2002).

${ }^{15}$ T. D. Bird, in GeneReviews, edited by R. A. Pagon, M. P. Adam, H. H. Ardinger, S. E. Wallace, A. Amemiya, L. J. Bean, T. D. Bird, C.-T. Fong, R. J. Smith, and K. Stephens (University of Washington, Seattle, 1993).

${ }^{16}$ M. Wojciechowska, K. Taylor, K. Sobczak, M. Napierala, and W. J. Krzyzosiak, RNA Biol. 11(6), 742 (2014).

${ }^{17}$ K. Michocka, D. Wieczorek, and R. Zieliński, Polish Patent Appl. P402717 (2013).

${ }^{18}$ C. E. Blanchet, A. Spilotros, F. Schwemmer, M. A. Graewert, A. G. Kikhney, C. M. Jeffries, D. Franke, D. Mark, R. Zengerle, F. Cipriani, S. Fiedler, M. Roessle, and D. I. Svergun, J. Appl. Cryst. 48(2), 431 (2015).

${ }^{19}$ A. Round, F. Felisaz, L. Fodinger, A. Gobbo, J. Huet, C. Villard, C. E. Blanchet, P. Pernot, S. McSweeney, M. Roessle, D. I. Svergun, and F. Cipriani, Acta Crystallogr., Sect. D 71, 67 (2015).

${ }^{20}$ P. V. Konarev, V. V. Volkov, A. V. Sokolova, M. H. J. Koch, and D. I. Svergun, J. Appl. Cryst. 36, 1277 (2003).
${ }^{21}$ M. V. Petoukhov, D. Franke, A. V. Shkumatov, G. Tria, A. G. Kikhney, M. Gajda, C. Gorba, H. D. T. Mertens, P. V. Konarev, and D. I. Svergun, J. Appl. Cryst. 45, 342 (2012).

${ }^{22}$ M. V. Berridge, P. M. Herst, and A. S. Tan, Biotechnol. Annu. Rev. 11, 127 (2005).

${ }^{23}$ J. H. Riazance, W. A. Baase, W. C. Johnson, K. Hall, P. Cruz, and I. Tinoco, Nucl. Acids Res. 13(13), 4983 (1985).

${ }^{24}$ J. Kypr, I. Kejnovská, D. Renčiuk, and M. Vorlíčková, Nucl. Acids Res. 37(6), 1713 (2009).

${ }^{25}$ Infrared Spectroscopy of Biomolecules, edited by H. H. Mantsch and D. Chapman (Wiley, 1996).

${ }^{26}$ S. Brahms, J. Brahms, and J. Pilet, Isr. J. Chem. 12(1-2), 153 (1974).

${ }^{27}$ J. F. Neault and H. A. Tajmir-Riahi, J. Biol. Chem. 272(14), 8901 (1997).

${ }^{28}$ G. J. Quigley and A. Rich, Science 194, 796 (1976).

${ }^{29}$ E. Taillandier and J. Liquier, Methods Enzymol. 211, 307 (1992).

${ }^{30}$ M. Banyay, M. Sarkar, and A. Gräslund, Biophys. Chem. 104(2), 477 (2003).

${ }^{31}$ E. Taillandier, J. P. Ridoux, J. Liquier, W. Leupin, W. A. Denny, Y. Wang, G. A. Thomas, and W. L. Peticolas, Biochemistry 26(12), 3361 (1987).

${ }^{32}$ M. Tsuboi, Appl. Spectrosc. Rev. 3(1) 45 (1970).

${ }^{33}$ H. Arakawa, J. F. Neault, and H. A. Tajmir-Riahi, Biophys. J. 81(3), 1580 (2001).

${ }^{34}$ H. A. Tajmir-Riahi, J. F. Neault, and M. Naoui, FEBS Lett. 370(1-2), 105 (1995).

${ }^{35}$ J. Prathiba and R. Malathi, Mol. Biol. Rep. 35(1), 51 (2008).

${ }^{36}$ J. F. Neault and H. A. Tajmir-Riahi, J. Phys. Chem. B 101(1), 114 (1997).

${ }^{37}$ See supplementary material at http://dx.doi.org/10.1063/1.4952589 for the deconvolution of characteristic absorption bands in FTIR spectra collected for LA12/siRNA lipoplexes (Fig. S1) and results of cytotoxicity tests of the LA12 lactose surfactant on the cell cultures (HeLa and fibroblasts GM04033 and GM07492) (Fig. S2).

${ }^{38}$ Z. Pietralik, Ż. Kołodziejska, M. Weiss, and M. Kozak, PLoS One 10(12), e0144373 (2015). 